\title{
Medición de variables psicológicas en deportistas de baile deportivo*
}

\section{Measure of Psychological Variables in Dancers Sport}

Recibido: noviembre 29 de 2010 | Revisado: julio 21 de 2011 | Aceptado: noviembre 12 de 2011

\author{
ENRIQUe CANTÓN ChIRIVELla** \\ Universidad de Valencia, España \\ IRENE CHECA ESQUIVA *** \\ Teseo Centro de Psicología, Valencia, España
}

SICI: 1657-9267(201209)11:3<921:MVPDBD>2.0.TX;2-Q

Para citar este artículo: Cantón, E. \& Checa, I. (2012). Medición de variables psicológicas en deportistas de baile deportivo. Universitas Psychologica, 11(3), 921-929.

" Artículo de investigación.

* Facultad de Psicología, Departamento de Psicología Básica. Av/ Blasco Ibáñez, №21, 46021, Valencia. E-mail: canton@uv.es. ResearcherID: Cantón, E. G-6118-2011.

**** $\mathrm{Pl}$ /Músico Ibars, 7 -esc.dcha- 3.a , 46920, Mislata, Valencia.E-mail: irecheca@hotmail.com

\section{RES U MEN}

El objetivo fundamental del estudio consiste en identificar y evaluar las principales variables psicológicas que están interviniendo en los distintos niveles de rendimiento en la práctica de baile deportivo. Para esto, entre otros, se utilizó el Cuestionario de Características Psicológicas aplicadas al Rendimiento Deportivo (Gimeno, Buceta \& Pérez-Llantada, 2001). Se realizó un análisis de varianza, y pese a no alcanzar las diferencias valores estadísticamente significativos en los factores Control del Estrés e Influencia de la Evaluación del Rendimiento, sí se puede señalar la existencia de diferencias relevantes en función de la interacción de dos condiciones: el nivel competitivo y el éxito deportivo, presentando tamaños del efecto relevantes $\left(n_{p}^{2}=0.230 ; n_{p}^{2}=0.164\right)$. Esta información ha sido la base para realizar un posterior trabajo aplicado, ajustado a las demandas de rendimiento en este deporte.

Palabras clave autores:

Psicología del deporte, baile deportivo, competición

Palabras clave descriptores:

Pruebas psicológicas, rendimiento deportivo, investigación cuantitativa.

\section{A B S T R A C T}

The aim of this study is to identify and assess key psychological variables are intervening at different levels of performance in sport dance practice. For this reason, among others, was used the "Cuestionario de Características Psicológicas aplicadas al Rendimiento Deportivo" (Gimeno, Buceta \& Pérez-Llantada, 2001). Carrying out an analysis of variance, although not reaching statistically significant differences in the factors Stress Management and Influence of Performance Measurement, if we can indicate the existence of relevant differences in terms of interaction two conditions: "Competitive level" and "sporting success" presenting relevant effect sizes $\left(n_{p}^{2}=0.230 ; n_{p}^{2}=0.164\right)$. This information is a basis for applied work adjusted to the demands of performance in this sport.

Key words authors:

Sport psychology, dancesport, competition.

Key words plus:

Psychological Tests, Performance Sport, Quantitative Research. 


\section{Introducción}

En los últimos años, cada vez son más los profesionales de la Psicología del Deporte y el Ejercicio que tienen la oportunidad de trabajar e investigar en deportes minoritarios o poco conocidos para el público en general (Díaz, 2010; Garcés de los Fayos, Pelegrín \& González, 2009). Llevar a cabo una intervención psicológica en este tipo de disciplinas deportivas exige realizar, en primer lugar, un estudio exhaustivo de las características de ese deporte, del que normalmente el profesional no suele tener conocimientos generales previos. Uno de estos casos es, precisamente, el que nos ocupa.

El baile deportivo es una disciplina que se practica en pareja mixta y propone la igualdad entre ambos sexos, ya que los bailarines masculinos y femeninos compiten delante de otras parejas, de forma simultánea y en la misma pista de competición. Además, la música es en esta disciplina una característica tan importante como lo pueda ser en el patinaje artístico, la gimnasia rítmica o la natación sincronizada (Eaton, 2006; Law \& Ste-Marie, 2005).

En competición existen dos modalidades: Baile Standard y Baile Latino. Cada una de ellas consta a su vez de cinco bailes distintos. Los Bailes Standard son el Vals Inglés, el Vals Vienés, el Tango Europeo, el Slow Fox y el Quickstep. En los Bailes Latinos nos encontramos con la Samba, el Cha-cha-cha, la Rumba-Bolero, el Pasodoble y el Jive. Existen deportistas que se especializan en una de las modalidades y otros que compiten en ambas.

Las competiciones de baile deportivo se desarrollan en pistas rodeadas por los jueces y con el público alrededor de las mismas y también en las gradas. Su cercanía y extensión supone para los deportistas una fuente constante de estímulos potencialmente distractores. El movimiento del propio cuerpo, la conexión con la pareja, los choques frecuentes contra contrincantes en pista, el ritmo de la música, el público, los jueces... Una buena gestión de la atención en cada momento significa un baile más coordinado y eficaz.

Siguiendo la ya clásica pero vigente clasificación de Riera (1985), podemos encuadrar el baile deportivo como un deporte de "cooperación", ya que es necesaria la acción conjunta de los dos miembros de la pareja, y debe ser considerado "sin oposición" ya que aunque las competiciones se desarrollan en el mismo espacio físico y los choques son frecuentes, las parejas no tienen como objetivo el llevar a cabo una oposición o interferencia directa en el rendimiento de las otras.

El baile deportivo es una disciplina cargada de un componente estético muy importante. Un gesto técnico bueno no tiene que estar únicamente "a ritmo", sino también bien expresado facial y corporalmente. Los deportistas deben también intentar llamar la atención de los jueces con su vestuario y su presentación estética, tanto con el maquillaje como con el peinado.

En cuanto al método de entrenamiento, también tiene características especiales. Los entrenamientos se basan en sesiones de la pareja con su entrenador en el que se crean y revisan los programas propios, se mejoran algunos gestos, se revisa el ritmo, etc. Pero además, requiere de muchos entrenamientos de repetición que debe llevar a cabo la pareja, por su cuenta, sin presencia del entrenador. Esta circunstancia hace que la relación de la pareja sea un componente vital para el rendimiento en pista y convierte en necesaria la habilidad de los deportistas para marcarse sus propios objetivos y ritmos sin las indicaciones directas de su entrenador. Estos objetivos deben ser consensuados entre los dos miembros de la pareja y su entrenador, y serán uno de los aspectos clave que se incluyan dentro del plan de trabajo psicológico.

Las competiciones se desarrollan en diferentes rondas (cuartos de final, semifinal, final) de aproximadamente 12 minutos de duración cada una, por lo que los deportistas pueden competir durante $4 \mathrm{o}$ 5 horas seguidas, con varios espacios de descanso intermedios, que pueden variar de entre 30 minutos a, incluso, varias horas. Además, el público y los jueces se sitúan al lado de pista rodeándola completamente, de pie o en sillas, a muy pocos metros de los propios deportistas. Todas estas características inherentes a la disciplina hacen que el trabajo focalizado en todas estas variables psicológicas sea importante, y para esto es imprescindible la evaluación previa. 
La medición de variables psicológicas es parte de la investigación, pero cuando se trabaja profesionalmente con deportistas es una de las tareas imprescindibles por parte del experto. De hecho, es consustancial al trabajo aplicado y muchos autores (Cantón, 2009; Dosil, 2002; Garcés de los Fayos, 2006; entre otros) han resaltado la importancia de la fase de evaluación dentro de una intervención bien planificada. Además, la utilización de instrumentos eficaces, bien adaptados y con unos índices adecuados de fiabilidad y validez, son algunos de los pilares básicos de la evaluación. Gracias a ellos, además de conseguir información de forma rápida y precisa, se obtienen datos relevantes que ofrecen una visión general de las características psicológicas de ese deporte en particular y de la posible orientación de la intervención.

Hay que tener en cuenta la necesidad de vincular claramente el conocimiento básico en Psicología con las necesidades o demandas especificas de una actividad competitiva particular y estas con una adecuada evaluación, lo que permitirá realizar una correcta intervención dentro de un plan de trabajo aplicado bien fundamentado y ajustado a la persona o grupo con el que se va a trabajar. Es importante, en primer lugar, poseer un conocimiento exhaustivo y especializado en Psicología (Cantón, 2010). Pero además, es importante poseer conocimientos que nos permitan comprender mejor la dinámica psicológica de un deporte particular y, por esto, el objetivo de este estudio es analizar cómo se comportan determinadas variables psicológicas en un grupo de deportistas de baile de competición.

\section{Hipótesis}

Tras la revisión de la literatura científica especializada y el análisis pormenorizado del deporte por parte del equipo de trabajo, conjuntamente con profesionales del sector, se elaboraron las siguientes hipótesis de investigación:

- El grupo de mayor nivel competitivo obtendrá puntuaciones significativamente mayores en las variables psicológicas medidas que el grupo de menor nivel competitivo. Las variables psicológicas medidas son los factores del CPRD: Con- trol del Estrés, Influencia de la Evaluación del Rendimiento, Motivación y Habilidad Mental. Es de esperar que aquellos deportistas con una técnica y exigencia mayores, y debido a los años de experiencia que han acumulado, obtengan mejores resultados en estas variables.

- El grupo que ha mejorado su rendimiento, es decir, aquellas parejas que han subido de categoría durante esta temporada, obtendrá puntuaciones significativamente mayores en las variables psicológicas medidas que el grupo que ha mantenido la categoría. Cabe plantear la hipótesis de que mejores puntuaciones en las variables psicológicas se comportan como predictores de mejores resultados deportivos. Creemos que mejores resultados en Control del Estrés, Influencia en la Evaluación del Rendimiento, Motivación y Habilidad Mental, se correlacionarán con mejores resultados deportivos.

\section{Método}

\section{Participantes}

El grupo esta compuesto de 19 deportistas pertenecientes al Club de Baile de Competición Stylo's Mislata (Valencia, España), con edades comprendidas entre los 11 y los 37 años. El grupo lo forman 10 deportistas hombres y 9 deportistas mujeres. Se obtuvo autorización explícita, tanto de los deportistas como de la entidad, para realizar la investigación aplicada y también para hacer público el nombre del centro deportivo.

Por otro lado, hemos elegido como variable de estudio la categoría de edad tal y como se estructura en la competición de baile, porque se consideró un dato más representativo que la edad cronológica. Una pareja de baile deportivo puede competir en un determinado rango de edad siempre que uno de los miembros cumpla el requisito de estar en ese rango de edad. Esto significa, que existen deportistas que compiten contra bailarines mayores, porque su pareja de baile sí cumple el requisito de la edad. Sin embargo, la pareja no podrá bailar en una categoría de edad menor si cualquiera de los dos miembros de la pareja supera la edad límite. En 
el grupo de estudio, 11 deportistas compiten en la categoría Adulto I y II (entre 18-35 años), cinco en categoría Youth (16-18 años) y tres en Junior I y II (entre 12 y 16 años).

En cuanto al nivel competitivo de las parejas del grupo de estudio, el perfil competitivo es de nivel medio-alto, ya que 11 de las parejas compiten en el nivel $\mathrm{A}$ o $\mathrm{B}$, que son las categorías más altas a nivel amateur, y 8 parejas compiten entre las categorías C y F. Las seis categorías de competición han sido distribuidas en dos grandes grupos por un motivo: hasta la categoría $\mathrm{C}$, las parejas no tienen programas libres e individualizados, es cuando ascienden a B cuando los programas técnicos se complican y son diferentes para cada pareja.

El grupo evaluado fue dividido también entre aquellos deportistas que habían ascendido de categoría o "letra", que fueron 10, y aquellos que habían mantenido la categoría, que sumaban nueve casos.

\section{Instrumentos}

Junto con reiteradas entrevistas y sesiones de observación, para la medición de las variables psicológicas que eran de interés en esta investigación, se utilizó el Cuestionario de Características Psicológicas aplicadas al Rendimiento Deportivo-CPRD que ha demostrado su fiabilidad y validez en varios estudios (Buceta, Gimeno \& Pérez-Llantada, 1994). Este instrumento de autoinforme valora cinco factores diferentes: Control del Estrés, Influencia de la Evaluación del Rendimiento, Motivación, Habilidad Mental y Cohesión de Equipo. El factor Control del Estrés indica los recursos que el deportista tiene para regular el estrés en competiciones deportivas, mientras que el factor Influencia en la Evaluación del Rendimiento está muy relacionado con el estrés también y específicamente con la ansiedad social o la evaluación inapropiada que el sujeto hace de su propio rendimiento. El factor Motivación mide características como la importancia del deporte respecto a otras actividades o la relación coste/beneficio de su práctica. El factor Habilidad Mental evalúa destrezas como el establecimiento de objetivos, la visualización, el análisis objetivo de rendimiento o las estrategias cognitivas. Por último, el factor
Cohesión de Equipo se refiere a la integración del deportista dentro del equipo o grupo deportivo. La consistencia interna del cuestionario es aceptable con un coeficiente alfa de 0.85 y los factores Control del Estrés, Influencia en la Evaluación del Rendimiento y Cohesión de Equipo también cuentan con índices de consistencia interna aceptables $(0.88$, 0.72 y 0.78$)$. Sin embargo, las escalas Motivación y Habilidad Mental presentan unos índices de consistencia interna débiles ( 0.67 y 0.34$)$.

Para el grupo objeto de esta investigación, se decidió extraer del cuestionario los ítems que saturaban en el factor Cohesión de Equipo, ya que se trata de una disciplina de pareja y con entrenamientos mayoritariamente sin la presencia del grupo.

\section{Procedimiento}

La evaluación de los deportistas fue llevada a cabo dentro del marco de una intervención profesional más amplia realizada durante las temporadas 2008/09 y 2009/10, y que continúa en la actualidad. Como se ha señalado, todos los deportistas y los entrenadores del club estaban informados del procedimiento que se iba a llevar a cabo y se realizó con el consentimiento de todos los participantes. La recogida de información mediante cuestionarios se realizó siempre bajo la supervisión de la psicóloga que informó previamente a los deportistas del anonimato de los resultados personales.

\section{Análisis de datos}

Los datos recogidos durante la evaluación fueron analizados mediante el paquete estadístico SPSS 17.0. Teniendo en cuenta los objetivos de la investigación y el reducido tamaño de la muestra $(N<30)$, se decidió aplicar una prueba de varianza que permitiera observar los efectos de cada condición y también de la interacción de ambas condiciones.

\section{Resultados}

Tras llevar a cabo en el análisis de la información obtenida, el primer dato interesante que se encontró se refiere a la correlación entre los factores del 
TABLA 1

Correlaciones entre las puntuaciones de los factores del CPRD

\begin{tabular}{lccccc}
\hline & Control del & $\begin{array}{c}\text { Influencia de la } \\
\text { evaluación del } \\
\text { estrés }\end{array}$ & Motimiento & $\begin{array}{c}\text { Habilión } \\
\text { mental }\end{array}$ \\
\hline Control del estrés & Correlación de Pearson & 1 & $0.78(* *)$ & -0.044 & 0.438 \\
& Sig. (bilateral) & $N$ & $<0.001$ & 0.857 & 0.061 \\
& $N$ & 19 & 19 & 19 & 19 \\
\hline Influencia de la & Correlación de Pearson & $0.78(* *)$ & 1 & -0.076 & 0.362 \\
evaluación del & Sig. (bilateral) & $<0.001$ & & 0.758 & 0.128 \\
rendimiento & $N$ & 19 & 19 & 19 & 19 \\
\hline Motivación & Correlación de Pearson & -0.044 & -0.076 & 1 & 0.237 \\
& Sig. (bilateral) & 0.857 & 0.758 & & 0.328 \\
& $N$ & 19 & 19 & 19 & 19 \\
\hline Habilidad mental & Correlación de Pearson & 0.438 & 0.362 & 0.237 & 1 \\
& Sig. (bilateral) & 0.061 & 0.128 & 0.328 & 19 \\
& $N$ & 19 & 19 & 19 & 19 \\
\hline
\end{tabular}

Fuente: elaboración propia.

propio instrumento empleado. Una correlación de medias (Tabla 1) muestra una $r=0.78$ con un nivel de significación $a=0.001$ entre los factores Control del Estrés e Influencia en la Evaluación del Rendimiento, tomando el total del grupo investigado. Sin embargo, entre los factores Motivación y Habilidad Mental, no se encontraron correlaciones significativas.

En cuanto a las hipótesis referidas a las diferencias en habilidades psicológicas entre bailarines de diferentes niveles competitivos y entre bailarines con diferentes resultados competitivos, se hallaron resultados interesantes. Una de las hipótesis planteadas en un principio se refería a la relación entre los grupos creados nivel competitivo y éxito deportivo y las puntuaciones en los diferentes factores del CPRD.

TABLA 2

Grupos de estudio por nivel competitivo y éxito

\begin{tabular}{ll}
\hline Grupos creados & Condiciones \\
\hline Nivel competitivo & Pertenecer a letra A o B \\
& Pertenecer a letra C, D, E o F \\
Éxito deportivo & Ascenso de categoría \\
& Permanencia en la categoría \\
\hline
\end{tabular}

Fuente: elaboración propia.
El grupo de estudio se dividió en dos, según el nivel competitivo: Grupo 1 que engloba a categoría A y B, y Grupo 2 que comprende las categorías C, D, E y F. Asimismo, también se conformaron en dos grupos empleando el criterio de éxito deportivo, siendo el Grupo A aquellos que ascienden de categoría y el Grupo B aquellos que permanecen en la misma categoría (Tabla 2).

La primera hipótesis sostenía que el grupo de mayor nivel competitivo obtendrá puntuaciones significativamente mejores en los factores del CPRD que el grupo de menor nivel competitivo. Y la segunda hipótesis planteaba que el grupo que ha mejorado su rendimiento durante esta temporada obtendrá puntuaciones significativamente mejores en los factores del CPRD que el grupo que ha mantenido la categoría.

Como se puede observar en la Tabla 3, no se encuentran diferencias significativas en el factor Control del Estrés del CPRD ni entre los dos grupos de nivel competitivo ni entre los dos grupos de éxito deportivo. Sin embargo, la interacción entre las dos variables independientes sí es significativa (tamaño del efecto relevante $>0.13$ ) pero no se ha captado la significación debido al reducido tamaño del grupo investigado. Es posible observar la relación entre las tres variables con la Figura 1. 
TABLA 3

Análisis de la varianza Control del Estrés

\begin{tabular}{lcccc}
\hline \multicolumn{1}{c}{ Origen } & $\mathrm{F}$ & Sig. & Tamaño del efecto & Potencia observada \\
\hline Modelo corregido & 1.672 & 0.216 & 0.251 & 0.349 \\
Intersección & 66.501 & $<0.001$ & 0.816 & 1 \\
Nivel & 0.186 & 0.673 & 0.012 & 0.069 \\
Éxito & 0.105 & 0.75 & 0.007 & 0.061 \\
Nivel * Éxito & 4.48 & 0.051 & 0.23 & 0.508 \\
\hline
\end{tabular}

Fuente: elaboración propia.

En el caso del factor del CPRD Influencia en la Evaluación del Rendimiento, se encontraron resultados similares a los obtenidos con el factor Control del Estrés. Los efectos principales no son significativos, ni con la variable nivel competitivo ni con la variable éxito deportivo. Pero sí se encontró un tamaño del efecto relevante en la interacción de las dos variables (0.164), aunque la potencia es reducida (0.363) debido al tamaño del grupo. En la Tabla 4 de datos y en la Figura 2, se muestra esta relación.

En los factores Motivación y Habilidad Mental, no se encuentran sin embargo diferencias significativas entre los diferentes grupos de nivel competitivo y de éxito deportivo ni en la interacción de los dos.

\section{Discusión}

Los resultados observados corresponden a un grupo de estudio pequeño de baile deportivo, una muestra de oportunidad, pero se consideró que dado que este tipo de actividad deportiva no había sido medida anteriormente, ofrece la posibilidad de conocer mejor este deporte y establecer algunas relaciones con variables psicológicas que pudieran orientar investigaciones posteriores. Asimismo, se pretendió destacar el interés por vincular la intervención profesional con la obtención de información útil y la investigación empírica.

Los resultados obtenidos con el análisis de datos ayudan a responder a las hipótesis planteadas. El primero de los resultados relevantes estaba plenamente relacionado con el método de evaluación que fue utilizado durante la intervención, el CPRD. La literatura acerca de este cuestionario (Pérez-Llantada et al., 2001; Gimeno, Buceta \& Pérez-Llantada, 2001) señala la relación directa que existe entre dos de sus factores: Control del Estrés e Influencia de la Evaluación del Rendimiento. Los autores del instrumento de evaluación señalan que este último factor se relaciona también con el estrés, pero que constituye un factor específico relacionado con la ansiedad social y con la interpretación inapropiada que hace el deportista de su propio rendimiento. Como muestran los datos obtenidos en este grupo de baile deportivo, los dos factores correlacionan de forma significativa y positiva ya que las personas que puntúan alto en Control del Estrés lo hacen también en Influencia de la Evaluación del Rendimiento.

TABLA 4

Análisis de la varianza de Influencia de la Evaluación en el Rendimiento

\begin{tabular}{lcccc}
\hline \multicolumn{1}{c}{ Origen } & $\mathrm{F}$ & Sig. & Tamaño del efecto & Potencia observada \\
\hline Modelo corregido & 1.152 & 0.360 & 0.187 & 0.249 \\
Intersección & 73.133 & $<0.001$ & 0.83 & 1 \\
Nivel & 0.144 & 0.71 & 0.01 & 0.065 \\
Éxito & 0.144 & 0.71 & 0.01 & 0.065 \\
Nivel * Éxito & 2.95 & 0.106 & 0.164 & 0.363 \\
\hline
\end{tabular}

Fuente: elaboración propia. 


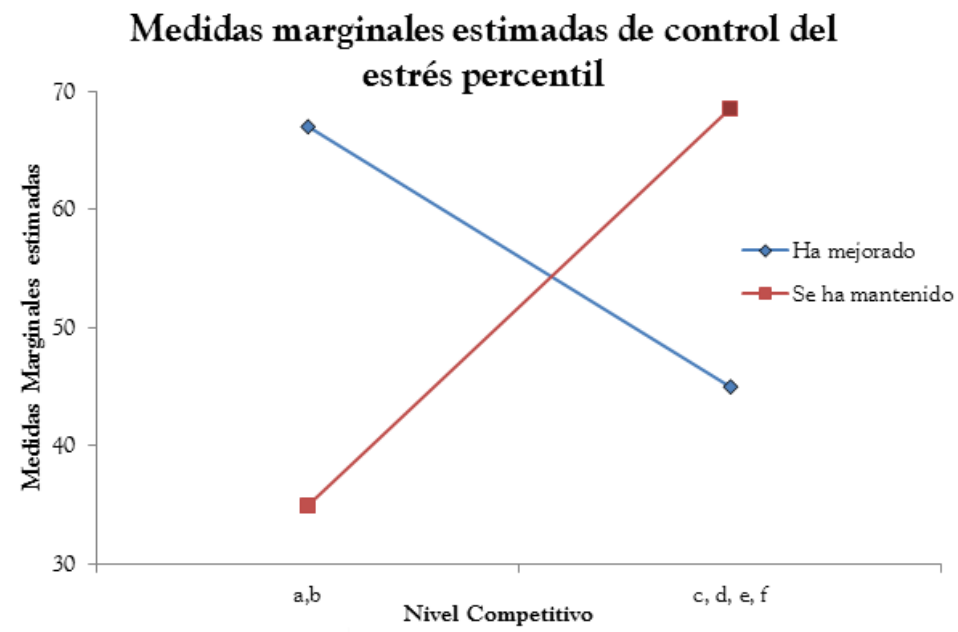

Figura 1. Interacción entre éxito deportivo y nivel competitivo en Control del Estrés.

Fuente: elaboración propia.

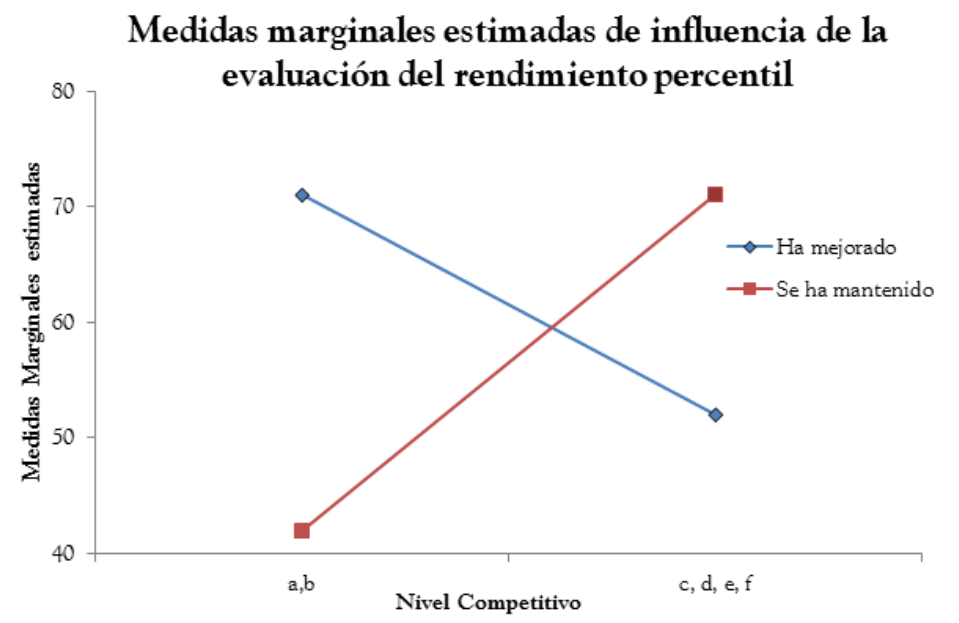

Figura 2. Relación éxito deportivo y nivel competitivo en Control del Estrés.

Fuente: elaboración propia.

La primera hipótesis planteaba que el grupo de mayor nivel competitivo obtendría puntuaciones significativamente mejores en los factores del CPRD que el grupo de menor nivel competitivo. Sin embargo, los datos rechazan esta hipótesis, ya que no se encontraron diferencias significativas en los factores del CPRD, salvo cuando se combinaron las dos condiciones creadas: éxito deportivo y nivel competitivo. Con la segunda hipótesis ha ocurrido algo similar: se formuló la hipótesis de que el grupo que ha mejorado su rendimiento durante esta tem- porada obtendría puntuaciones significativamente mejores en los factores del CPRD que el grupo que ha mantenido la categoría. Sin embargo, como en la hipótesis anterior, no se encontraron diferencias significativas entre los dos grupos si se tiene en cuenta únicamente la adscripción a uno de ellos. Se entiende que es muy probable que la falta de significación en las dos hipótesis pueda deberse, principalmente, al tamaño del grupo.

Si bien es cierto que las dos hipótesis anteriores resultaron no significativas estadísticamente, los 
resultados ofrecen otros datos interesantes. Cuando se tienen en cuenta, tanto el nivel competitivo, como el éxito deportivo durante esta temporada, se encuentra que estas dos variables interrelacionadas sí ofrecen diferencias significativas en las puntuaciones de los factores Control del Estrés e Influencia de la Evaluación del Rendimiento.

El grupo de mayor nivel competitivo que ha mejorado su rendimiento esta temporada obtiene resultados significativamente más altos en las dos dimensiones enunciadas que el grupo que ha mantenido la categoría. Sin embargo, en el caso del grupo de menor nivel competitivo se encuentran resultados opuestos: el grupo que ha mejorado su rendimiento obtiene resultados significativamente menores en las dos dimensiones del CPRD que el grupo que ha mantenido la categoría.

La explicación a estos resultados puede plantearse teniendo en cuenta diferentes aspectos. Por un lado, es cierto que para el ascenso de categoría son necesarias cinco victorias en las que influyen multitud de factores que no tienen que ver con variables psicológicas. Una de las explicaciones puede tener que ver con el instrumento de evaluación empleado. El CPRD es un cuestionario orientado a la competición y que está cargado de situaciones relacionadas con un nivel competitivo alto. Es posible que el grupo de mayor nivel competitivo sepa gestionar mejor las variables relacionadas con el estrés y haya conseguido el ascenso, mientras que los deportistas de nivel competitivo menor, con menor experiencia, no dominen todavía las habilidades relacionadas con niveles tan altos de competición. En todo caso, y como una apreciación cualitativa consecuencia del trabajo aplicado que se sigue llevando a cabo en la escuela, las habilidades psicológicas consiguen dotar a todos los deportistas, sin tener en cuenta su experiencia, de recursos psicológicos potencialmente útiles.

Parece que los resultados señalan, una vez más, que las aptitudes psicológicas son más importantes en la carrera deportiva conforme la exigencia competitiva aumenta. Diferentes autores (Cantón, Checa \& Ortín, 2009; Cruz, 1995; entre otros) han encontrado que la habilidad para manejar las variables psicológicas tiene habitualmente más peso en los resultados conforme el deportista aumenta de categoría y/o nivel competitivo en cualquier deporte. Por esto, los planes de intervención deben estar diseñados según el nivel competitivo y la edad de los deportistas, y evitar intervenciones sistematizadas no adaptadas a la realidad del deportista.

Cabe destacar que este estudio tiene en cuenta no solo valores de significación estadística, sino también los datos del tamaño del efecto, como demandan algunos autores en los últimos años (Frías, Pascual \& García, 2000). Se reconoce que, debido al tamaño de la muestra, los valores de significación y de potencia se ven afectados, por lo que el tamaño del efecto supone una fuente fiable de relación entre variables.

En cuanto a las limitaciones del estudio, es necesario resaltar en primer lugar, el tamaño de la muestra. Si bien también es cierto que el número de deportistas de baile de competición en el país es reducido si se compara con otros deportes. Con todo, se consideró que sería deseable llevar a cabo este estudio con un número mayor de sujetos o con más grupos de deportistas para ver el radio en que los resultados son o no similares.

Este estudio se enmarca dentro de una intervención diseñada e implementada para esta escuela en particular, pero se cree que estos datos cuantitativos contribuyen a guiar el trabajo de los profesionales que quieran trabajar en esta disciplina.

\section{Referencias}

Buceta, J. M., Gimeno, F. \& Pérez-Llantada, M. C. (1994). Cuestionario de Características Psicológicas relacionadas con el Rendimiento Deportivo (CPRD). Universidad Nacional de Educación a Distancia (sin publicar).

Cantón, E. (2009). La profesión de psicólogo del deporte. Revista de Psicología General y Aplicada, 62, 121-130.

Cantón, E. (2010). La psicología del deporte como profesión especializada. Papeles del Psicólogo, 31(3), 237-245.

Cantón, E., Checa, I. \& Ortín, F. J. (2009). Intervención psicológica con un nadador de medio acuático abierto. Cuadernos de Psicología del Deporte, 9(2), 57-65. 
Cruz, J. (1995). Psicología del deporte. Madrid: Síntesis.

Díaz, J. (2010). El entrenamiento mental de los jugadores de bolos: preparación para el alto rendimiento. Torrelavega, Cantabria: Federación Cántabra de Bolos.

Dosil, J. (2002). Psicólogo del deporte: asesoramiento e intervención. Madrid: Síntesis.

Eaton, L. (2006). Mental training por shynchronized swimming pool. Synchro Swimming USA, 14(1), 7.

Frías, M. D, Pascual, J. \& García, J. F. (2000). Tamaño del efecto del tratamiento y significación estadística. Psicothema, 12(2), 236-240.

Garcés de los Fayos, E., Pelegrín, A. \& González, J. (2009). Entrenamiento psicológico aplicado a diferentes modalidades deportivas. En J. Díaz, I. Díaz \& J. Dosil (Eds.), La Psicología del deporte en Iberoamérica (pp. 150-154). Torrelavega: SIPD.

Garcés de los Fayos, E. (2006). Evaluación psicológica en contextos deportivos. En E. J. Garcés de los Fayos,
A. Olmedilla \& P. Jara (Coords.), Psicología y deporte (pp. 429-446). Murcia: Diego Marín.

Gimeno, F., Buceta, J. M. \& Pérez-Llantada, M. C. (2001). El cuestionario Características Psicológicas relacionadas con el Rendimiento Deportivo CPRD: características psicométricas. Análise Psicológica, 1(19), 93-113.

Law, B. \& Ste-Marie, D. M. (2005). Effects of self-modelling on figure skating jump performance and psychological variables. European Journal of Sport Science, 5(3), 143-154.

Pérez-Llantada, M. C., Buceta, J. M., López de la Llave, A., Gimeno, F. \& Ezquerro, M. (2001). El cuestionario Características Psicológicas relacionadas con el Rendimiento Deportivo (CPRD): un estudio con la escala de control del estrés. Revista Electrónica de Motivación y Emoción, 5, 11-12.

Riera, J. (1985). Introducción a la psicología del deporte. Barcelona: Editorial Ariel. 
